REVISÃO DE LITERATURA: "A INFLUÊNCIA DO

TRATAMENTO COM ANÁLOGO DE GNRH NA AL-

TURA FINAL ANTES E APÓS A PUBERDADE PRE-

COCE

\title{
LITERATURE REVIEW: "THE INFLUENCE OF \\ TREATMENT WITH GNRH ANALOG IN THE FINAL HEIGHT BEFORE AND AFTER EARLY PUBERTY "
}

Naiara Roberta Ribeiro de Marins ${ }^{1}$

Lorriene Reis Branquinho de Carvalho Ferreira ${ }^{2}$

Lilian Maria Soares ${ }^{3}$

Resumo: A puberdade precoce (PP) se inicia com a ativação do eixo gonadotrófico antes dos 8 anos em meninas e aos 9 anos em meninos . Uma das principais preocupações é a altura final pois essa acelera o processo de maturação óssea resultando em uma estatura reduzida. $\mathrm{O}$ tratamento padrão para a puberdade precoce consiste no uso de depósitos de análogos de GnRh com a proposta de minimizar os efeitos da puberdade precoce, melhorar a altura proposta final e psicológica .Em virtude disso, essa revisão de literatura se fundamenta em demostrar os efeitos da utilização dos análogos de GnRh na altura final antes e após os 8 anos

\begin{tabular}{ll}
\hline 1 & UNIBRASIL \\
2 & UNIBRASIL \\
3 & UNIBRASIL
\end{tabular}


de idade.

Palavra-Chave: Puberdade precoce, Análogo de GnRH, Altura.

Abstract: Earl puberty begins with activation of the gonadotrophic axis before 8 years of age in girls and at 9 years of age in boys. One of the main concerns is the height because it accelerates the bone maturation process resulting in reduced height. The standard treatment for precocious puberty is the use of deposits of GnRh analogues with the proposal to minimize the effects on growth. As a result, this literature review is based on demonstrating the effects of using $\mathrm{GnRh}$ analogues at the final height. Before and after 8 years of age.

Keyword: Puberty preocoe, GnRH analog, Height

\section{INTRODUÇÃO}

A puberdade precoce é provocada pela ativação do eixo hipotálamo - hipófise - gonadal antecipadamente em meninas e meninos, aos 8 e 9 anos. Esse processo, pode ser ocasionado por lesões orgânicas no sistema nervoso central, alterações genéticas ou idiopáticas, acometendo cerca de $95 \%$ do gênero feminino por causas idiopática, e $50 \%$ dos meninos são por lesões orgânicas. Tais alterações, resultam no comprometimento da estatura final em decorrência do fechamento precoce da placas epifisárias de crescimento. O diagnóstico pode ser feito através de exames laboratoriais e de imagem. O tratamento consiste em utilizar drogas para o bloqueio puberal são os análogos de GnRh.

Em virtude disso, essa revisão de literatura tem como

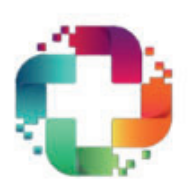


ISSN: 2763-5724

Vol. 01 - n 02 - ano 2021

Editora Acadêmica Periodicojs

objetivo demonstrar os efeitos pubarca sem o avanço do cres-

do tratamento com análogos de cimento e da idade óssea. Tanto

GnRH na estatura final, ao ini- a puberdade precoce verdadeira

ciar antes e após os oito anos de (PPV) como a pseudo puberdaidade.

de precoce devido ao aumento dos esteroides sexuais, por atuar

METODOLOGIA

nas adrenais, gônadas e tireoide, pode levar a uma baixa estatura

Essa revisão de lite- final esperada em comparação ratura foi embasada nas fontes com outras crianças da mesma de pesquisa da Google scholar, faixa etária e sexo.

Scielo e Pubmed .

Dentre as causas da puberdade precoce podemos citar as RESULTADO E DISCUSSÃO lesões orgânicas no sistema nervoso central, os defeitos genétiA puberdade precoce é cos, ou idiopáticos. A puberdade um conjunto de patologias que precoce central (PPC) raramente engloba as variantes normais do acontece apresentando incidêncrescimento, adrenarca e telar- cia de $1: 5.000-1: 10.000$. De ca precoce, e alterações físicas. acordo com a distribuição por gêHabitualmente, a idade inicial nero sexo entre meninas e menipara o desenvolvimento puberal nos é esperado uma proporção de adequado é de nove anos em me3:1 e 23:1. As causas idiopáticas ninos e oito anos nas meninas. e orgânicas representam, $98 \%$ e Muitas desenvolvem sinais pu$69 \%$ em meninas e 0 a $60 \%$ em berais isolados, como a telarca, meninos, respectivamente.

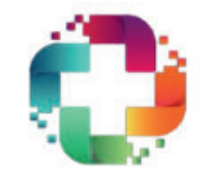


ISSN: $2763-5724$

Vol. 01 - n 02 - ano 2021

Editora Acadêmica Periodicojs

O avanço desigual da nos meninos, o aumento dos va-

maturação óssea decorrentes do fechamento antecipado da cartilagem de crescimento, reduz a estatura final. O bloqueio da liberação das gonadotrofinas, na idade adequada e para os com PPC, resulta no bloqueio da liberação dos esteroides gonadais sendo capaz de prevenir ou restaurar o déficit da estatura.

Em decorrência disso, faz se necessário o diagnostico da PP que é clínico e laboratorial. Nesse, é fundamental se pautar no surgimento dos caracteres sexuais secundários, a velocidade de progressão e os estágios de Tanner. A curva de crescimento é indispensável, pois a velocidade de crescimento, geralmente, apresenta aumento para faixa etária contribuindo para ajudar na conduta terapêutica. Os exames laboratoriais, consistem nas dosagens dos esteroides sexuais lores da testosterona é útil para avaliação da PP; já o estradiol é restrito, pois este hormônio oscila seus valores e pode ter níveis baixos mesmo com a PPC; a dosagem de Gonadotrofinas é importante para diferenciar se a origem é central ou periférico. $\mathrm{O}$ “padrão-ouro" para o diagnostico da PPC é o teste de estimulação com GnRH. Outros hormônios podem ser dosados adicional: como andrógenos adrenais, função tireoidiana, $17 \mathrm{OH}$ progesterona e determinação de hCG podem ser fundamentais para descobrir causas secundárias. Os exames de imagens, são: Ultrassonografia pélvico (a medida longitudinal do útero e o seu volume são os parâmetros mais fidedignos da puberdade). A idade óssea é determinada através do método de Tanner - Whitehouse (TW-20) para 20 núcleos das mãos e pu-

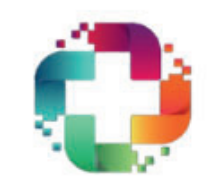


ISSN: 2763-5724

Vol. 01 - n 02 - ano 2021

Editora Acadêmica Periodicojs

nhos tem boa sensibilidade para o diagnóstico puberdade precoce.

A ressonância magnética é importante para avaliar a etiologia da PPC. Os pacientes, de forma geral, devem realizar uma avaliação por imagem da área hipotalâmica hipofisária.

A estatura final resulta do diagnóstico e tratamento antecipado. Na puberdade precoce central (PPC), é introduzir hormônios que atuariam bloqueando a liberação de LH, FSH e assim gerar ganho adicionais na estatura final e melhora muitas vezes do ganho do fato psicossocial.

A terapêutica utilizada no protocolo de puberdade precoce GnRH-dependente faz uso de análogos agonistas hiperativos do GnRH (GnRHa) de liberação lenta (depot).

Estes medicamentos, em poucos dias, reduzem os números de receptores do GnRH (down-regulation) nos gonado-

trofos hipofisários, causando a dessensibilização dos receptores por deslocamento do sinal de transdução intracelular.

Em decorrência disso, os análogos do hormônio liberador de gonadotrofina (GnRHa) são as medicação de escolha para melhorarem a estatura final em crianças com PPV. Os melhores resultados foram obtidos quando a idade de crescimento é inferior a seis anos de idade e até aos oito anos de idade porém com menor predileção ao avanço da estatura final comparado com menores de seis anos de idade. O tratamento de meninas após estas idades com puberdade precoce, todavia, não apresentou beneficio da altura final observada em relação a estatura prevista no inicio do tratamento.

Através disso, conclui que não houve diferenças impor-

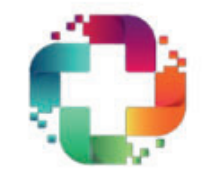


tantes nos escores de desvio padrão da altura final (SDS) e SDS da altura inicial ao comparar o GnRHa e os grupos de controle de puberdade antes e após os oito anos de idade.

\section{CONSIDERAÇÕES FINAIS}

O uso de hormônios análogos de GnRH para o tratamento da puberdade precoce verdadeira com ênfase na estatura final, apresentou eficácia em idade inferior a seis anos de idade comparado a administração após os oito anos de idade, que não teve resultados significativos na estatura final, entretanto apresentou resultados dependentes da hereditariedade e outros fatores que interferem na obesidade. Ambas as faixas etárias tiveram em comum um beneficio satisfatório na parte psicológica, que contribuíram para o tratamento.

\section{REFERÊNCIAS:}

BEREKET, Abdullah. A Critical Appraisal of the Effect of Gonadotropin-Releasing Hormon Analog Treatment on Adult Height of Girls with Central Precocious Puberty. 2017. 16 f., J Clin Res Pediatr Endocrinol, İstanbul, 2017. Disponível em: http://cms. galenos.com.tr/Uploads/Article_16628/JCRPE-9-2-En.pdf. Acesso em: 5 abr. 2021.

PASQUINO, Anna Maria; PUCARELLI, Ida; SEGNI, Maria; MATRUNOLA, Marco; CERRONE, Fabio. Altura adulta em meninas com puberdade precoce central tratadas com análogos do hormônio liberador de gonadotrofina e hormônio do crescimento. 1999. 4 f., Journal Of Clinical Endocrinology And Metabolism, Roma, 1998. Disponível em: ht- 
ISSN: 2763-5724

Vol. 01 - n 02 - ano 2021

Editora Acadêmica Periodicojs

tps://academic.oup.com/jcem/ article/84/2/449/2864152. Acesso em: 5 abr. 2021.

RAMOS, Carolina de Oliveira. Avaliação de pacientes com puberdade precoce central após o tratamento com análogos de GnRH: aspectos antropométricos, metabólicos, reprodutivos e psicossociais. 2019. 168

f., Universidade de Sao Paulo, Sao Paulo, 2019. Disponível em: https://www.teses.usp.br/ teses/disponiveis/5/5135/tde06072020-163149/publico/CarolinadeOliveiraRamosVersaoCorrigida.pdf. Acesso em: 5 abr. 2021.

HANNA, Carolina Macedo Saidah. Hiperplasia congênita da suprarrenal versus puberdade precoce verdadeira: uma visão panorâmica na infância. 2019. 73 f. , Universidade Federal de Goiás, Goiania, 2019. Disponível em: https://repositorio.bc.ufg.br/tede/ handle/tede/10334. Acesso em: 5 abr. 2021.

LANES, Roberto; SOROS, Arlette; JAKUBOWICZ, Salomon. Formas de puberdade acelerada versus lentamente progressiva em meninas com puberdade precoce e precoce. Efeito supressor da gonadotrofina e altura final obtida com dois análogos diferentes. 2004. 17 v., Ournal Of Pediatric Endocrinology \& Metabolism, Londres, 2004. Disponível em: https://pubmed.ncbi. nlm.nih.gov/15237711/\#article-details. Acesso em: 5 abr. 2021.

LI, Pin; LI, Yan; YANG, Chung-Lin. Tratamento com agonista do hormônio liberador de gonadotrofina para aumentar a estatura final em crianças com puberdade precoce: uma meta-análise. 2014. 93 v, Xangai, 2014. Disponível 
ISSN: 2763-5724

Vol. 01 - n 02 - ano 2021

Editora Acadêmica Periodicojs

em: https://journals.lww.com/

md-journal/Fulltext/2014/12020/

Gonadotropin_Releasing_Hor-

mone_Agonist_Treatment.52. aspx. Acesso em: 5 abr. 2021.

SILVA, Ana Cláudia C.s. da; ADAN, Luís Fernando F. Crescimento em Meninos e Meninas Com Puberdade Precoce. 2003. 10 f., Sociedade Brasileira de Endocrinologia e Metabologia, Salvador, 2003. Disponível em: https://www.scielo.br/pdf/abem/ v47n4/a14v47n4.pdf. Acesso em: 5 abr. 2021.

CAREL, Jean-Claude et al. Puberdade precoce e crescimento estatural. 2004. 10 v., Human Reproduction Update, Paris, 2004. Disponível em: https:// academic.oup.com/humupd/article/10/2/135/617162. Acesso em: 5 abr. 2021.
BENETTI-PINTO, Cristina Laguna et al. Fatores determinantes do ganho na altura em meninas com puberdade precoce central idiopática tratadas com análogo de GnRH. 2008. 1 f., Revista Brasileira de Ginecologia e Obstetrícia, Rio de Janeiro, 2008. Disponível em: https://www.scielo.br/scielo.php?script=sci_arttext\&pi032008001200004\&1n$\mathrm{g}=\mathrm{pt} \& n \mathrm{~nm}=\mathrm{iso} \& \operatorname{lng}=\mathrm{pt}$. Acesso em: 5 abr. 2021.

MONTE, Osmar; LONGUI, Carlos Alberto; CALLIARI, Luis Eduardo P.. Puberdade Precoce: Dilemas no Diagnóstico e Tratamento. 2001. 45 v., Arquivos Brasileiros de Endocrinologia \& Metabologia, Sao Paulo, 2001. Disponível em: https://www.scielo.br/scielo.php?script=sci_arttext\&pi 7302001000400003\&ln$\mathrm{g}=\mathrm{pt} \&$ tlng=pt. Acesso em: 5 abr. 2021. 
ISSN: 2763-5724

Vol. 01 - n 02 - ano 2021

Editora Acadêmica Periodicojs

Disponível em: https://www.

PUCARELLI, Ida; SEGNI, Maria; ORTORE, Massimiliano; MORETTI, Lessandra; IANNACCONE, Riccardo; PAS QUINO, Anna Maria. Terapia combinada com análogo de GnRH mais hormônio de crescimento na puberdade precoce central. 2000. 13 v., Journal Of Pediatric Endocrinology And Metabolism, Londres, 2000. Disponível em: https://www.degruyter.com/document/doi/10.1515/ JPEM. $2000.13 . \mathrm{S} 1.811 / \mathrm{htm} 1$. Acesso em: 5 abr. 2021.

TUNG, Yi-Ching; LEE, Jing-Sheng; TSAI, Wen-Yu; HSIAO, Pei-Hung. Os efeitos da terapia análoga ao hormônio liberador de gonadotrofina em meninas com puberdade precoce dependente de gonadotrofina. 2007. 106 v., Journal Of The Formosan Medical Association, Taiwan, 2007. sciencedirect.com/science/article/pii/S0929664608600479?via\%3Dihub. Acesso em: 5 abr. 2021.

MONTE, Osmar; LONGUI, Carlos Alberto; CALLIARI, Luis Eduardo P.. Puberdade Precoce: Dilemas no Diagnóstico e Tratamento. 2001. 45 v., Arquivos Brasileiros de Endocrinologia \& Metabologia, Sao Paulo, 2001. Disponível em: https://www.scielo.br/scielo.php?script=sci_arttext\&pi302001000400003\&1n$\mathrm{g}=$ pt\&tlng=pt. Acesso em: 5 abr. 2021.

KOHN, Brenda; JULIUS, Joanne R.; BLETHEN, Sandra L.. Uso combinado de hormônio do crescimento e análogos do hormônio liberador de gonadotrofina: a experiência do estudo cooperativo nacional de crescimento. 1999.

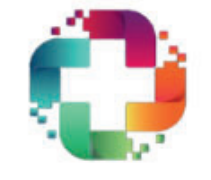


ISSN: 2763-5724

Vol. 01 - n 02 - ano 2021

Editora Acadêmica Periodicojs

6 f., Academia Americana de

Pediatria, Estados Unidos, 1999.

Disponível em: https://pediatrics.

aappublications.org/content/pe-

diatrics/104/Supplement_5/1014.

full.pdf. Acesso em: 5 abr. 2021. 